\title{
CARACTERÍSTICAS DE UN GRUPO DE MUJERES EMBARAZADAS QUE SE SOMETIERON VOLUNTARIAMENTE A LA PRUEBA DE VIH EN EL HOSPITAL SAN RAFAEL DE FACATATIVÁ
}

\section{CHARACTERISTICS OF A GROUP OF PREGNANT WOMEN VOLUNTARILY TESTED FOR HIV ANTIBODIES IN THE FACATATIVA'S SAN RAFAEL HOSPITAL}

\author{
William Onatra H. ${ }^{1}$ \\ Álvaro Cuervo ${ }^{2}$ \\ Myriam Castañeda ${ }^{3}$ \\ Fernando Charry ${ }^{4}$ \\ Sandra Barreto ${ }^{5}$ \\ Angélica Moreno ${ }^{4}$ \\ Edisson Marín ${ }^{4}$
}

\begin{abstract}
RESUMEN
A nivel mundial, el principal factor de riesgo de contagio en la mujer en la adquisición del VIH son las relaciones sexuales. Prevenir la trasmisión vertical madre-hijo es el objetivo del programa y, con este estudio, se deseaba conocer la prevalencia de VIH en mujeres embarazadas, en el municipio de Facatativá. En la consulta externa de control prenatal, se buscaron las madres con factores de riesgo para VIH y SIDA, quienes recibieron una asesoría y una evaluación pre-test. Se registraron 620 mujeres embarazadas que se sometieron voluntariamente a la prueba de VIH; el 65\% eran menores de 24 años. De los

${ }^{1}$ Médico Ginecólogo. Docente de Ginecología y Obstetricia U.D.C.A Carrera 9 No 48-51 Con. 206. Bogota. Colombia.wonatra@gmail.com

${ }^{2}$ Médico Epidemiólogo. Subgerente Hospital San Rafael de Facatativá (HSRF)

3 Enfermera Jefe de Consulta Externa HSRF

${ }^{4}$ Grupo de Médicos Especialistas. Epidemiólogo. Psicología, Consulta Externa HSRF.

${ }^{5}$ Estadística Universidad de Ciencias Aplicadas y Ambientales U.D.C.A
\end{abstract}

620 casos registrados, el $84,35 \%$ tenía seguridad social. Solo dos de ellas salieron positivas, una en la contra muestra negativa. A los casos positivos, se les aplicó la prueba confirmatoria de Western Blot. Se ejecutó el protocolo propuesto de AZT por cuatro semanas antes del parto. Después de éste la madre recibió nueva asesoría, se suprimió la lactancia y se le ofreció lactancia artificial por doce meses. Con una prevalencia del 0,06\%, las principales características de la población mostraron: el promedio de la menarquia fue de 12,9 años, el inicio de la relaciones sexuales estaba por debajo de los 16 años (44\%), el $50 \%$ no tuvo orientación sexual y solo fue obtenida en el colegio (36\%). Su primer embarazo ocurrió por debajo de los 22 años. Este programa permite confirmar que la prueba durante control prenatal detecta madres $\mathrm{VIH}$ positivas y su manejo adecuado previene la infección vertical madre-feto.

Palabras clave: VIH, SIDA, embarazo, adolescencia.

\section{SUMMARY}

At world-wide level, sexual intercourse is the main risk factor for women to be infected by $\mathrm{VIH}$. Prevention of vertical mother-child transmission is the objective of a 
program and, with this study, we wanted to determine the prevalence of $\mathrm{VIH}$ in pregnant women in the municipality of Facatativá. In the external consultation of prenatal control, we looked for women with risk factors for $\mathrm{VIH}$ and AIDS; they received advisory and a pretest evaluation was performed. 620 voluntary pregnant women were registered and underwent the $\mathrm{VIH}$ test; $65 \%$ were younger than 24 years. Of the 620 cases $84.35 \%$ had social security. Only two were found to be positive, later, one showed to be negative in the contra test. The positive cases were confirmed by the Western Blot test. The protocol proposed by AZT was applied during fore weeks before childbirth. After the childbirth the mother again received advices, the lactation was suppressed and artificial lactation implemented during twelve months. With a prevalence of $0.06 \%$, the main characteristics of the population showed: the average of menarche was of 12.9 years; the initiation of sexual relations was before the age of 16 years (44\%); $50 \%$ of the women had not received sexual orientation and only $36 \%$ had it obtained at school. The first pregnancy happened before the age of 22 years. This program confirms that the prenatal test detects positive $\mathrm{VIH}$ mothers and its adequate treatment prevents the vertical infection mother-foetus.

Key words: HIV, AIDS, pregnancy, adolescence

\section{INTRODUCCIÓN}

De acuerdo al programa de la Organización de las Naciones Unidas (ONU) sobre el Virus de la Inmunodeficiencia (VIH), ONUSIDA (2004) calculó que para julio del 2004 habían en el mundo 37,8 millones de personas con VIH-Sida, de los cuales 17 millones eran mujeres, 18,7 millones hombres y 2,1 millones menores de 15 años. El $67 \%$ viven en el África y el 5,4\% en América Latina y el Caribe. Se estima que 630 menores de 15 años son infectados anualmente, mueren $490.000,90 \%$ en el África. En América Latina, se presentan 140.000 nuevos casos por año y 65.000 mueren (ONUSIDA, 2006). La proporción de mujeres infectadas que en 1997 era del 41\%, para el 2004 aumentó al 50\% (García et al. 2005). Los casos colombianos son superados por Brasil y México y su prevalencia se ha calculado en 0,6-0,7\% que corresponde a unas 58.000 mujeres en edad fértil. Un estudio de transmisión madre-hijo muestra que de 200.853 pruebas en mujeres embarazadas, 377 con VIH, para una probabilidad de transmisión de 0,19\%; 285 tuvieron algún tipo de tratamiento, de quienes nacieron doce niños infectados (García et al. 2005). El predominio era mayor en hombres para 1998, proporción de 13:1, al aumentar la relación heterosexual en ambos sexos para el 2004, fue de 3:1 (Prieto, 2005). La prevalencia es baja en Venezuela y Chile, con el $2 \%$ y alta en Argentina, Colombia y Brasil con el 6\%. La prevalencia en Colombia es del 3,3\% en Barranquilla, Santanderes y Quindio y de un $4 \%$ en trabajadoras sexuales (ONUSIDA, 2006).

Dado que el diagnóstico y manejo oportuno del VIH durante el embarazo previene la contaminación del recién nacido, se han postulado varios esquemas de diagnóstico y terapéutica. Recientemente, se han introducido algunas modificaciones en el desarrollo de intervenciones eficaces y asequibles que reducen la probabilidad de que una mujer transmita el VIH a su hijo (Fonseca E Prieto, 2005). Las tres intervenciones más importantes para las mujeres $\mathrm{VIH}$ positivas son: el suministro de fármacos anti-retrovirales tipo zidovudina (ZDV), adecuada atención del parto (cesárea) y la supresión de la lactancia (Wade et al. 2004). Se exige, en estos casos, que la mujer sepa que esta infectada, que necesita un asesoramiento y pruebas que permitan conocer su estado respecto al $\mathrm{VIH}$. Los servicios de asesoría y de pruebas con relación al embarazo en los diferentes servicios de salud pueden constituir una puerta de entrada valiosa para la prestación del servicio a mujeres sanas y asintomáticas, así como a su pareja (Ministerio de la Protección Social, 2000). Las asistencias voluntarias no se limitan a prevenir la transmisión materno infantil del $\mathrm{VIH}$ sino que serán una fuente de apoyo e información. Las pruebas de VIH, se han utilizado como diagnóstico para confirmar el SIDA sintomático. Diferentes estudios confirman que la asesoría y las pruebas voluntarias en poblaciones grandes potencian los comportamientos seguros a nivel individual reduciendo la ignorancia, el miedo y el estigma de estar infectado (CDCP, 2001). Se propone en los diferentes centros de salud una oficina de asesoría a la mujer embarazada y que en los casos de $\mathrm{VHH}$ positivos, se les brinde un apoyo psicológico, farmacológico y de seguimiento al recién nacido (Shetty \& Maldonado, 2001).

A medida que aumenta el contagio, se incrementa la posibilidad de infección durante el embarazo que se realiza por tres mecanismos: a) transmisión in útero, donde a veces se ha identificado $\mathrm{VIH}$ en el tejido fetal a la octava semana de que representa el $40 \%-80 \%$ y es causada por el contacto fetal con la sangre y el líquido amniótico. c) transmisión por la leche materna, causa en un 14\%-30\% (Fowler et al. 2000). Tan rápido como mueren las células 
infectadas son reemplazadas, hasta que sobreviene una disfunción inmunitaria para hacer ineficaz el control del virus, llegando progresivamente a las condiciones clínicas que definen el SIDA (Kuhn et al. 2000).

Desde la publicación del Pedriatrics AIDS Clinical Trials Group (PACGT 076) (Camparelli et al. 2003), diversos estudios mostraron que la terapia antirretroviral reducía la transmisión vertical de VIH madre-hijo inicialmente con zidovudina (ZDV) como monoterapia, después con dos y posteriormente con tres fármacos. En Colombia, el Sistema de Seguridad Social en Salud (SGSSS) no ha logrado implantar la cobertura total alcanzando solo el $66 \%$ debido a las restricciones y las dificultades financieras.

El presente estudio pretendió evaluar la frecuencia de VIH-SIDA en mujeres gestantes que asistieron a control prenatal en el Hospital General de Facatativá, prestar asesoría y manejo a los casos sero-positivos y de esta forma prevenir la transmisión vertical madre feto. Hace parte del proyecto "Reducción de la transmisión MadreHijo del VIH en Colombia", auspiciado por el programa conjunto de la ONU sobre el VIH-SIDA (ONUSIDA), el INS y la Fed. Col.de Coop. de Hospitales (Min. Prot. Soc, 2003; García et al. 2005).

\section{MATERIALES Y MÉTODOS}

Se trata de un estudio prospectivo de prevalencia para detectar madres embarazadas $\mathrm{VIH}(+)$ y de prevenir la transmisión vertical al feto aplicando el Protocolo propuesto por ONUSIDA. En la consulta externa de control prenatal, se buscaron las madres con factores de riesgo para $\mathrm{VIH}$ y SIDA, tales como inicio de relaciones tempranas, diferentes compañeros sexuales, drogadicción, antecedentes de tatuajes, violación. Las pacientes seleccionadas recibieron asesoría y un pre-test por parte del Departamento de Enfermería Sección de Prevención y Promoción del hospital. Previo consentimiento, se tomaron y se remitieron las muestras de sangre diariamente al laboratorio de referencia de la Secretaría de Salud de Bogotá.

Como método de tamizaje, se utilizó, la técnica de inmunoabsorción ligada a enzimas (ELISA) - ultramicroelisa Tecnosuma y para la confirmación de la infección, la técnica de Western blot (Murex HIV de Abbott) aceptando como positivos las recomendaciones
CDC de Atlanta (CDCP, 2001). La prueba de ELISA utiliza antígenos derivados del virus íntegro y los une a recipientes de micro-titulación. Se coloca el suero o plasma de la paciente en los recipientes y si hay anticuerpos contra $\mathrm{VIH}$, se unen al antígeno presente. Se proporciona, además, un sustrato cromógeno para la enzima potencialmente unida y se lee la intensidad del color que se genera en un espectrofotómetro. La lectura del color es proporcional a la cantidad del complejo unión enzima-anticuerpo adherido al antígeno de $\mathrm{VIH}$ que se encuentra en el recipiente (Endres, 1996). Las pruebas que se disponen en el comercio tienen tasas de sensibilidad y especificidad entre 98 y 100\%. Se aplicó la técnica de Western Blot. Mediante la comparación de la localización de las bandas con las de testigo es posible determinar si el suero de la paciente contiene anticuerpos específicos para VIH (Celum et al. 2001).

El protocolo propuesto para las madres $\mathrm{VIH}$ positivas confirmadas indica la administración de Zidovudina $300 \mathrm{mg}$ vía oral cada $12 \mathrm{~h}$ durante 15 días antes del parto, cesárea, suspensión de la lactancia y suministrar leche complementaria al recién nacido (ONUSIDA 2002). Los casos seropositivos fueron evaluados por el Departamento de Obstetricia y Ginecología del Hospital San Rafael de Facatativá, los cuales recibieron el AZT cuatro semanas antes del parto. El parto, se programó para cesárea electiva. La Universidad de Ciencias Aplicadas y Ambientales U.D.C.A apoyó el programa con un docente y los estudiantes rotantes por el servicio de Obstetricia y Ginecología encargados de las visitas domiciliarias. Después del parto, la madre recibió nueva asesoría por parte del Departamento de Enfermería, se suprimió la lactancia y se le practicó el seguimiento al recién nacido, de acuerdo al protocolo. El departamento de Pediatría del Hospital evaluó cada tres y seis meses a estos niños con muestras seriadas para VIH, de acuerdo a la resolución 412 del Ministerio de Salud. El departamento de Trabajo Social evaluó el ambiente socio-económico familiar y de su entorno.

Los formularios de recolección incluían: fecha ingreso, dirección, edad, ocupación, seguridad social, estado civil, factores de riesgo (antecedentes de enfermedades sistémicas y de transmisión sexual ETS), menarquia, ciclos, última regla, semanas de embarazo, número de gestaciones, partos o abortos, inicio de relaciones sexuales, resultados de la prueba, registro de la asesoría y consentimiento informado; para los casos positivos, protocolo terapéutico: fecha de la cesárea, fecha 
de controles al recién nacido y datos de laboratorio confirmatorio de $\mathrm{VIH}$. Los datos recolectados, se registraron en una base de Epi-info y se utilizaron pruebas paramétricas y no paramétricas. La probabilidad de transmisión madre e hijo, se relacionó con el efecto de la carga viral pre-parto y la obtenida en el recién nacido. Este proyecto piloto fue auspiciado por ONUSIDA Colombia, INS, FEREDSALUD con cobertura nacional, donde participó el Hospital San Rafael de Facatativá con la Coordinación de las Secretarías de Salud de Cundinamarca y de Bogotá,

\section{RESULTADOS Y DISCUSIÓN}

Información general: Del total de 620 mujeres embarazadas que aceptaron la prueba de VIH-SIDA durante la consulta prenatal del Hospital, se obtuvieron los siguientes resultados: un promedio de edad entre 18 y 22 años (Gráfica 1). La mayoría de ellas tienen Servicio de Seguridad en Salud beneficiando 406 casos (65,48\%); por parte de la Asegurada de Riesgos (ARS), 117 casos (18,87\%); un número alto de 116 desplazados (18,7\%). No sabe o no responde 97 , casos para un $15,65 \%$. En

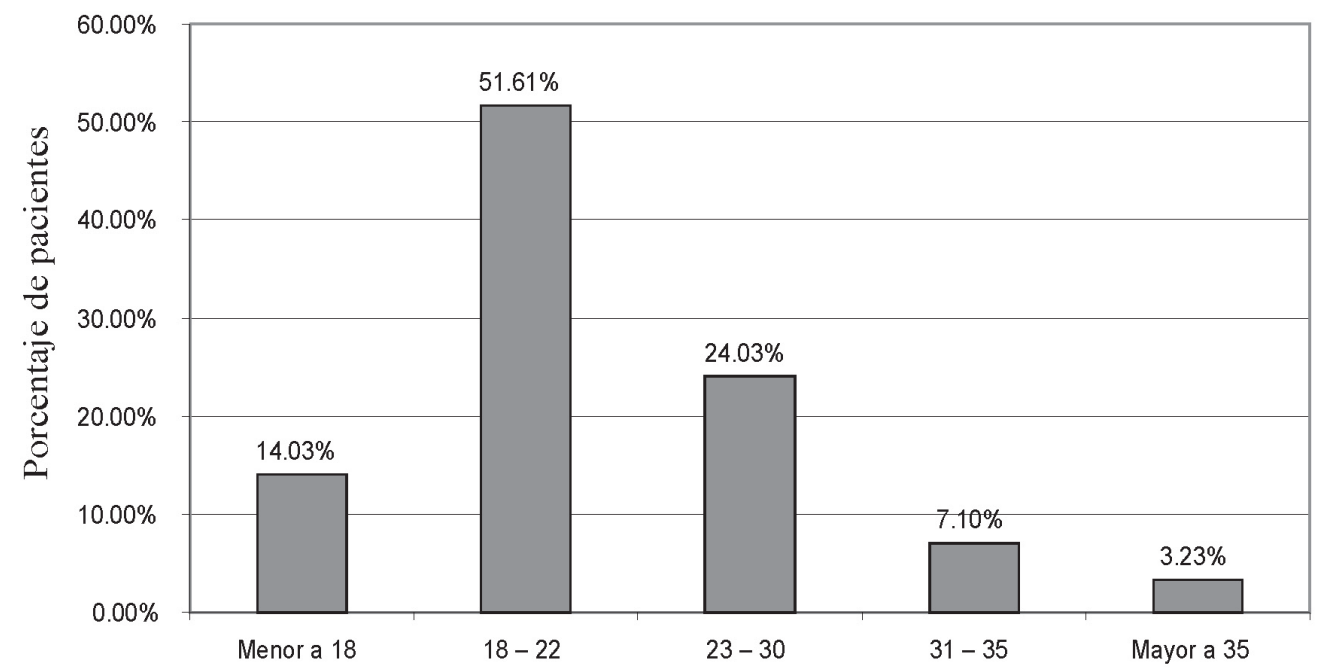

Gráfica 1. Porcentaje de promedio de edad de mujeres embarazadas en consulta prenatal.

un estudio con 200.853 pruebas en mujeres embarazas en Colombia (García et al. 2005), se detectaron 337 gestantes positivas, con promedio de edad de 24 años. De las 620 pruebas, 486 recibieron asesoría pre-test y pos-test y 134 se sometieron a la prueba sin evaluación previa. Del total de los exámenes realizados dos fueron positivas con una contra-muestra negativa (Tabla 1). El caso positivo, se manejó con el protocolo propuesto por el Ministerio de la Protección Social (2003). La prevalencia, de acuerdo al estudio nacional, es de 0,19\%, los departamentos con mayor porcentaje son Quindío $0,67 \%$, Guajira $0,63 \%$, Santander $0,48 \%$ y Cordoba $0,64 \%$ (García et al. 2005).

Tabla 1. Selección de pacientes.

\begin{tabular}{|l|c|c|c|c|c|c|}
\hline \multicolumn{1}{|c|}{ SELECCIÓN } & SI & $\%$ & NO & $\%$ & Ns/NR & $\%$ \\
\hline Información & 486 & 78,39 & 4 & 0,65 & 130 & 20,97 \\
\hline Asesoria & 486 & 100 & & & & \\
\hline Prueba & 486 & 100 & & & & \\
\hline Prueba positiva & 2 & & & & & \\
\hline Prueba confirmatoria & 1 & & & & & \\
\hline
\end{tabular}


Referente al estado civil, se encuentran en unión libre 312 casos (50,3\%), no sabe o no responde $119(19,2 \%)$, separadas $106(17,1 \%)$, solteras 46 casos $(7,4 \%)$ y casadas un $6 \%$. En comparación, a nivel nacional, de 337 embarazadas positivas, el $72 \%$ presentaban unión estable y el $27 \%$ son solteras. A nivel de ocupación, la mitad de ellas, un $79,2 \%$ viven en el hogar, empleada, 81 casos (13,1\%); cesante 52, (8,4\%); 51 estudiantes $(8,2 \%)$ y un caso de desempleada $(0,16 \%)$; mientras que de acuerdo a García et al. (2005), el 90\% son amas de casa y un $4 \%$ trabajadoras sexuales.

En cuanto a los factores de riesgo, se encontraron con antecedentes de tatuaje 102 casos; el resto, infecciones de transmisión sexual, dos casos $(0,3 \%)$, trasfusiones
12 casos $(1,94 \%)$, accesorios percútanos (pircing), uno, para un $0,2 \%$. Como antecedentes de enfermedades sistémicas llama la atención que todas obedecen a procesos virales (gráfica 2).

Antecedentes ginecológicos: El promedio de la menarquia para el grupo fue de 12,9 años, encontrándose menos de los 11 años, un 2,6\%; entre los 11 y 13 años, un $49,7 \%$; entre los $14-18$ años, un $28,7 \%$ y no sabe o no responde, el $19,03 \%$. Con relación a la orientación sexual no existe en un 40,97\%, no sabe o no responde el 20,6\% y solo hay información en el colegio en 184 casos (30\%). Del resto la información obtenida es muy pobre: en un centro de salud (3,71\%), la EPS/ARS y en charlas $1,94 \%$, y en el hogar, un $1,13 \%$.

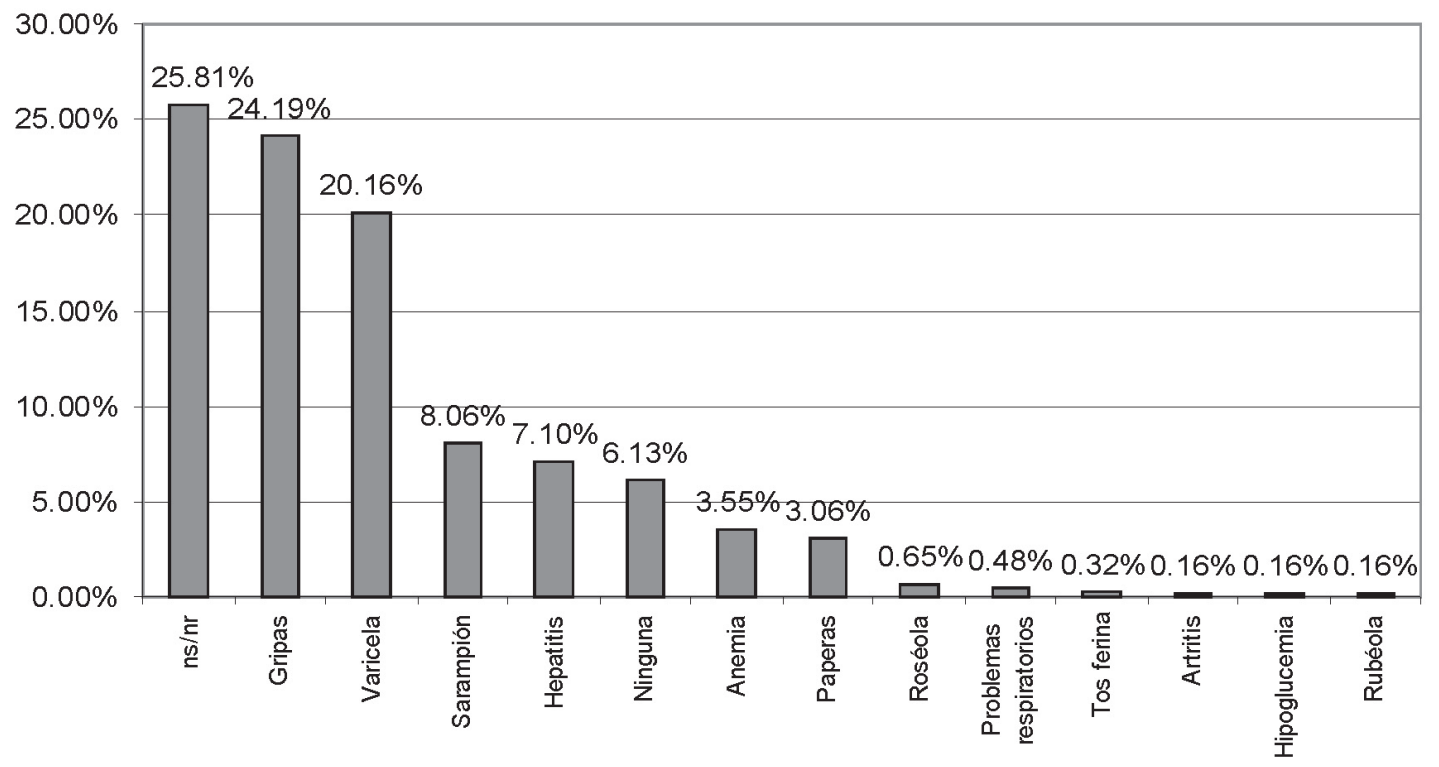

Gráfica 2. Porcentaje de antecedentes de enfermedades sistémicas.

El inicio de las relaciones sexuales estuvo por debajo de los 16 años en el $44 \%$, lo cual explica el promedio de embarazos a esta edad y el desconocimiento de la sexualidad en este grupo. Solamente un $8,87 \%$ inicia las relaciones a una edad mayor de 19 años y como dato curioso, se obtuvo que el 20,32\% respondió: no sabe, no recuerda. En general es un grupo joven, con un primer embarazo en el $20 \%$ de los casos, antes de los 18 años.

A nivel mundial, el principal factor de riesgo de contagio en la mujer en la adquisición del VIH son las relaciones sexuales, ya que más del $90 \%$ de las mujeres infectadas por el VIH/SIDA en los países en desarrollo han contraído la infección por transmisión heterosexual (ONUSIDA, 2004). En la mayoría de los casos, estas mujeres tenían relaciones sólo con su pareja estable lo que avala la vulnerabilidad de ellas al contagio, el cual está en directa relación con el comportamiento de su pareja (bisexualidad, drogadicción, promiscuidad) (Urrea-Giraldo et al. 2006). Este resultado contrasta con el estudio de García et al. (2005), el cual mostró que de 337 mujeres embarazadas positivas, el $4 \%$ eran trabajadoras sexuales.

En América Latina, se estima que 1,4 millones de personas viven con VIH/SIDA y que 150.000 adquirieron la infección 
durante el año 2000, siendo la principal modalidad de contagio, al igual que en Chile, la exposición homosexual, observándose un crecimiento sostenido de la transmisión heterosexual, relegando a un tercer lugar la vía sanguínea y la de la transmisión vertical, esta última estimada en un 1,5\% del total de los casos, excluyendo a aquellos sin diagnóstico definitivo (pérdida de seguimiento de 27 niños) (Pérez E Dabis, 2003; Mor et al. 2006).

Son varias las razones que explican el por qué la mujer es más susceptible de adquirir la infección por VIH que el hombre (Stringer $\varepsilon$ Vermund, 1999). Dentro de estos, se deben conocer: a) La transmisión sexual del virus es varias veces mayor del hombre a la mujer que viceversa, presentando mayor riesgo aquellas mujeres que presenten una mucosa vaginal adelgazada o desprovista de mecanismos fisiológicos de defensa (púberes, posmenopáusicas). b) Vaginosis asintomática de transmisión sexual, las que frecuentemente no son diagnosticadas, alterando la identidad de la mucosa vaginal. c) Dependencia al hombre económica, social y emocionalmente, lo que le dificulta el rechazo a tipos de relaciones sexuales de alto riesgo. d) Pautas culturales que alientan la promiscuidad en el varón, lo que aumenta el riesgo de contagio en las monógamas y e) Falta de fácil acceso a métodos preventivos controlados por la mujer. Todo lo anterior avala la importancia de la educación de la sexualidad humana a todo nivel poblacional, proporcionando la información necesaria y reconociendo el derecho de la mujer a informarse para así lograr que ella tome las medidas apropiadas para protegerse y prevenir la infección por el VIH (OPS, 1999).

VIH y embarazo: Estudios epidemiológicos sugieren que las tasas de embarazos en mujeres seropositivas que no han presentado SIDA son comparables con aquellas no infectadas, mientras que las que desarrollaron la enfermedad la probabilidad de embarazo es bastante menor. La mayoría descubren su enfermedad durante el embarazo o posterior al parto al detectarse la infección en su descendencia. Con relación a la necesidad de hacer un tamizaje a todas las mujeres en su consulta pre y/o post-concepcional, ACOG (2000) recomienda realizarlo de forma rutinaria, conducta adoptada por el Ministerio de Protección Social. ONUSIDA Colombia (2004), obliga el acto de consejería pre-test, la necesidad de consentimiento informado y la voluntad en la decisión de la realización del examen, por lo que su toma requiere la participación de personal adiestrado en "consejería", la cual ha reforzado la decisión en la mujer a realizarse el examen y ha llevado a un significativo aumento en la adhesión a conductas preventivas y al tratamiento. Respecto a la Consejería pre y post-concepcional, es decir un "diálogo confidencial" entre una consultante y un consejero(a) para habilitar a la mujer para el enfrentamiento del estrés, el conocimiento del VIH-Sida sobre el embarazo y la discusión de elementos para la prevención de la transmisión vertical, son útiles (Prieto, 2005).

Afortunadamente, el estado gravídico no parece alterar la evolución o la historia natural de la infección por $\mathrm{VIH}$, ya que si bien es cierto se ha descrito un descenso del recuento de células CD4 durante el embarazo, éstas vuelven a su línea basal después del parto (Burns et al. 1999). En ausencia de complicaciones, tales como drogadicción o enfermedad médica crónica, la incidencia de patología obstétrica no aumenta permaneciendo el aborto espontáneo, restricción del crecimiento intrauterino, parto de pretérmino y muerte fetal dentro de prevalencias esperables. Sin embargo, en gestantes con un compromiso significativo del sistema inmunitario (CD4 $<300 / \mathrm{dl}$ ), el riesgo de las complicaciones infecciosas se incrementa (Minkoff et al. 1999).

Según los resultados del DANE, la población menor de 20 años corresponde al $20 \%$ del total, es decir, 8.438.847 personas aproximadamente, de las cuales el $50 \%$ corresponde a mujeres con un factor de riesgo para embarazo, embarazo no deseado y aborto. La encuesta Nacional de Demografía y Salud mostró que 44,5\% de las mujeres entre 20 y 24 años informaron haber tenido relaciones sexuales antes de los 18 años y el 59,5\% entre los 20 y 49 años (Profamilia, 2000); en el presente estudio, se confirma una edad más temprana, con un $47,3 \%$ con relaciones por debajo de los 16 años y un $4 \%$ por debajo de los 11 años. Esos datos explican el grave problema en los hogares, los colegios y el entorno social que vive la sociedad colombiana.

El inicio cada vez más temprano de relaciones íntimas aumenta también el contagio de infecciones de transmisión sexual (ITS) y, el VIH-SIDA y abortos. El $10 \%$ de los embarazos a nivel mundial ocurren en adolescentes, es decir, 13 millones de adolescentes dan a luz en los países en desarrollo (The Internationationl Perinatal HIV Group (TIP-HIV-G), 1999). ONUSIDA (2002), informa que en el mundo 11,8 millones de jóvenes entre 15 y 25 años (7,3 mujeres y 4,5 hombres) y en América Latina 560.000 (mujeres 31\% y hombre $69 \%$ están infectados por el virus. La disminución de 
las cifras en Europa muestra el cumplimiento de metas de prevención (Kourtis et al. 2006).

En las características generales, se aprecia el 30\% de este grupo vive en área rural y, de alguna manera, el 100\% posee seguridad social. La mayoría, $66,7 \%$, vive en unión libre y son solteras el $26,4 \%$. En contraste con la encuesta de PROFAMILIA (2000), que reporta unión libre $22 \%$ y $70 \%$ solteras. Los resultados muestran una frecuencia de $\mathrm{VIH}$ y embarazo dentro de su promedio con relación a otros datos para la población de Cundinamarca en menos del $1 \%$, sin olvidar las cifras nacionales y regionales donde la proporción de adultos que tuvieron dos o más parejas son del 33\% en hombres y 3,3\% en mujeres. Las regiones con más prevalencia masculina son Atlántico y Antioquia y menor, la Oriental. En la mujeres, la mayor prevalencia esta en la zona Pacífica, Central y Antioquia y menor, Atlántica y Oriental (PROFAMILIA, 2000).

Si bien los factores de riesgo están relacionados con las infecciones de transmisión sexual (ITS), relaciones sexuales no vaginales, tabaquismo, alcoholismo, uso de jeringas compartidas, transfusiones y tatuajes, en el presente estudio, el $6 \%$ acusa haber tenido una ITS, mientras que en la encuesta de Profamilia hay desconocimiento de lo que son la ITS, en un $28 \%$. No hay antecedentes de transfusiones o relaciones no vaginales. Solo aparece el tatuaje en un $18 \%$, cuando la relación en otros países es del $28 \%$. Los datos de la encuesta muestra que solo el $2 \%$ de los hombres y $1 \%$ de las mujeres, en el último año han sufrido de una ITS (Landesman et al. 1996).

Entre los antecedentes de enfermedades previas existe un desconocimiento en un $25 \%$; las que más recuerdan son la hepatitis, la varicela y la gripe. La edad de la menarquia promedio de 12,8 años está de acuerdo con varias encuestas en nuestro medio. Un 3,1\% la tiene por debajo de once años y explica la aparición de embarazos a tan corta edad y que ha movido a las autoridades de salud y rectores de colegios a tomar medidas preventivas en vista del aumento de embarazos durante la adolescencia. El inicio de las relaciones sexuales para este grupo es muy temprano; cerca del $85,3 \%$ ya han tenido la primera relación sexual antes de los 16 años, cuando el promedio nacional es de 15,8 años para los hombres y 18,9 para la mujeres. Este promedio ha descendido para la generación de 1992 que era de 16,8 años, para el 1994 ha disminuido a 15,3 años (PROFAMILIA, 2000). De las mujeres que acudieron a la consulta, ya el $41,1 \%$ habían tenido un hijo.
Terapia anti-retroviral en el embarazo: La meta de la terapia durante el embarazo es reducir la carga viral materna a niveles indetectables sin provocar efectos deletéreos o teratogénicos en el producto y disminuir el riesgo de la transmisión vertical (Stringer $E$ Vermund, 1999). Para lograr este objetivo, actualmente existen 14 medicamentos antirretrovirales disponibles, las cuales se deben emplear dentro de esquemas que sean individualizados para cada paciente, elección que se debiera basar en el tratamiento previo de la mujer, estado actual de la paciente y su motivación, carga viral y su resistencia a medicamentos, recuento de CD4, recomendándose la utilización de la Zidovudina(ZDV) dentro del esquema, ya que ha sido la única droga protocolizada que ha demostrado su eficacia en proteger al feto de la transmisión vertical (The Petra Study Team, 2002). Sin embargo, en nuevos estudios, otros antiretrovirales han demostrado también eficacia en reducir esta transmisión (Saba, 1999; Guay et al. 1999).

En Chile, utilizando el PACGT 076 protocolo, se puso en marcha formalmente desde 1996, obteniendo su financiamiento estatal con una cobertura del $100 \%$ de la demanda tanto del sector público como privado, siendo evaluado por CONASIDA (2005), durante este año 2002, alcanzando una tasa de transmisión global de 5,6\%, lo que significa una reducción de la transmisión histórica (30\%) de 81,3\% (Valdés, 2002).

Según la literatura internacional, la terapia antiretroviral se debería ofrecera toda gestante seropositiva sintomática y/o aquella asintomática que presente un recuento de CD4 menor a $500 \mathrm{cel} / \mathrm{mL}$, una carga viral mayor de 10.000 (bDNA) o mayor de 20.000 (RTPCR), siendo controversial el manejo para pacientes con un recuento de CD4 mayor de $500 \mathrm{cel} / \mathrm{mL}$ y cargas virales menores de 10.000 (bDNA) y 20.000 (RT-PCR). Otros esquemas son útiles en disminuir el riesgo de la transmisión, es así que la aleación de ZDV+ Lamivudina (Combivir) indicado durante el parto y en el postparto ha reducido el contagio en un 38\% (Saba, 1999), mientras que el Nevirapine, indicado de la misma manera, redujo la transmisión en un 50\% (Guay et al. 1999).

El meta-análisis realizado por Brocklehurst $\mathcal{E}$ Volmink (2002) evidencia que cualquier régimen de ZDV versus placebo reduce considerablemente el riesgo de transmisión, $46 \%$. Se disminuye el riesgo de muerte infantil dentro del primer año de vida en un $57 \%$ y riesgo de muerte materna en un $32 \%$. No existen evidencias que 
la AZT tenga alguna influencia en el parte pre-término o de bajo peso al nacer. En un estudio hecho en Colombia (García et al. 2005) realizando 200.853 pruebas y detectando 377 gestante infectadas $(0,19 \%)$, se aplica el protocolo de AZT 15 antes de parto y la probabilidad del transmisión vertical madre-hijo fue de $1,78 \%$.

Respecto a la vía del parto existe suficiente evidencia que la operación cesárea electiva disminuye aproximadamente a la mitad la probabilidad de contagio al recién nacido (The European Mode of Delivery Collaboration, 1999). Debe ser indicada, a las 38 semanas, para evitar la ocurrencia de patología perinatal, que aumente el riesgo de transmisión, como lo es el parto de pretérmino y la rotura prematura de membranas y, ante la eventualidad que ésta última ocurriera, la interrupción por vía alta se debería realizar antes de las cuatro horas de ocurrida (ACOG, 2000). Otras de las medidas recomendadas es la suspensión de la lactancia materna, ya que variadas publicaciones demostraron un riesgo global para la transmisión vertical en un 15\% en la ausencia de terapia anti-retroviral (Rouet et al. 2004; Breastfeeding and HIV International Transmission Study Group, 2004).

\section{RECOMENDACIONES}

Tamizaje precoz de infección por VIH: se debe ofrecer la opción a toda mujer que desee embarazo y/o en su inicio de embarazo, la realización de la serología para $\mathrm{VIH}$, previa consejería y consentimiento informado.

Terapia anti-retroviral: cuyo objetivo es reducir la carga viral y con ello prevenir en la gestante la progresión del $\mathrm{VIH}$ y la transmisión vertical, tres semanas antes de terminar el parto (Orio et al. 2007).

Drogas antiretrovirales en el embarazo: (Iribarren et al. 2001)

- En el esquema, no se deben usar Hidroxiurea ni Efavirenz (teratogénicos). No se debe indicar el Indinavir durante el tercer trimestre por el riesgo de hiperbilirrubinemia y cálculos renales en el recién nacido. Los inhibidores de las proteasas, potencialmente aumentan el riesgo de hiperglicemia. La Zidovudina (ZDV), el Epivir (3TC) y la Nevirapina son las únicas drogas con estudio farmacocinétic en mujeres embarazadas (Fonseca E Prieto, 2005; Zamorano \& Hirsch, 2004).

Protocolo de Manejo: Aceptar las recomendaciones y el protocolo propuesto por ONUSIDA -Ministerio de la Protección Social (ONUSIDA 2006).
Prevención: Continuar con el apoyo a las instituciones que desarrollan programas de prevención en ITS en adolescentes (OPS 1999).

Agradecimientos: A las directivas de la Universidad de Ciencias Aplicadas y Ambientales, a la Vicerrectoría de Investigaciones de la U.D.C.A por su asesoría, Directivas del Hospital San Rafael de Facatativá, al equipo humano y técnico del Área de Prevención y Promoción. Secretaría de Salud de Cundinamarca, Secretaría de Salud de Bogotá, D.C. por su dirección y apoyo logístico. Al Ministerio de la Protección Social por su proyecto multicéntrico auspiciado por ONUSIDA, INS, FEREDSALUD y al equipo de trabajo.

\section{BIBLIOGRAFÍA}

ACOG. 2000. American College of Obstetricians and Gynecologist. Committee on Obstetric Practice Sheduled cesarean delivery and prevention of vertical transmission of HIV infection. Committee Opinión. p.234.

BLOCKLEHURST, P.; VOLMINK, J. 2002. Antiretrovirals for reducing the risk of mother to child transmission of HIV infection. Cochrane Database Syst. Rev.2:CD003510

BREASTFEEDING AND HIV. INTERNATIONAL TRANSMISSION STUDY GROUP. 2004. Late postnatal transmission of HIV-1 in breast-fed children: and individual patient data meta-analysis. J. Infect. Dis. 189:2154-2166.

BURNS, D.N.; NOURJAH, P.; WRIGHT, D.J.; MINKOFF, H.; LANDESMAN, S.; RUBINSTEIN, A.; GOEDERT, J.J.; NUGENT. 1999: Changes in immune activation markers during pregnancy and postpartum. J. Reprod. Inmunol. 42:147-165 .

CAMPARELLI, E.V.; MIROCHNICK, M.; WAYNE M, DANKNER WM, BLANCHARD S, MOFENSON L MCSHERRY GD, GAY H, CIUPAK G, SMITH B, CONNOR JD; Pediatric aids clinical trials group 331 investigators. 2003. Pediatrics AIDS clinical trials group. Pharmacokinetics and tolerance of zidovudine in preterm infants. J. Pediatr. 142:47-52.

CDCP. 2001 Centers for Disease Control and Prevention. Revised guidelines for HIV counseling, testing and referral. MMWR Recom. Rep. 50:1-57. 
CELUM, C.L.; BUCHBINDER, S.P.; DONNELL, D.; DOUGLAS, J.M. Jr.; MAYER, K.; KOBLIN, B.; MARMOR, M.; BOZEMAN, S.; GRANT, R.M.; FLORES, J.; SHEPPARD, H.W. 2001. Early human immunodeficiency virus (HIV) infection in the HIV Network for Prevention Trials Vaccine Preparedness Cohort: risk behaviors, symptoms, and early plasma and genital tract virus load. J. Infect. Dis.183:23-35.

CONASIDA. Comision Nacional de SIDA. 2005. Ministerio de salud de Chile. Epidemiology of HIV/AIDS in Chile. Rev Chilena Infectol. 22:169-202.

ENDRES, M.J. 1996. CD4 independent infection by HIV-2 is mediated by fusin CXCR-4. Cell. 87:745-756.

FOWLER, M.G.; SIMOND, R.J.; ROONGPISUTHIPOONG, A. 2000. Update on perinatal HIV transmission. Pediatr. Clin. North Am. 47:21-38.

FONSECA, C.E.; PRIETO, F.E. 2005. Manejo de la infección materna con VIH y del recién nacido expuesto. Rev. Col. Obstet. Ginecol. 56:68-91.

GARCÍA, R.; PRIETO, F.; ARENAS, C.; RINCÓN, J.; CAICEDO, S.; REY, G. 2005. Reducción de la transmisión madre-hijo del VIH en Colombia: dos años de experiencia nacional 2003-2005. Biomedica 25:547-564.

GUAY, L.A.; MUSOKE, P.; HOM, D.L.; NAKABIITO, C.; BAGENDA, D.; FLETCHER, C.V.; MARUM, L.H.; FOWLER, M.G.; FALKSVEDEN, L.G.; WAHREN, B.; KATAAHA, P.; WIGZELL, H.; MMIRO, F.A.; JACKSON, J.B. 1999. Intrapartum and neonatal single dose nevirapine compared with AZT for prevention of mother to child transmission of HIV-1 in Kampala Uganda: HIVET 012 randomized trial. Lancet 354:795-802.

IRIBARREN, J.A.; RAMOS, J.T.; GUERRA, L.; COLL, O.; DE JOSE, M.I.; DOMINGO, P.; FORTUNY, C.; MIRALLES, P.; PARRAS, F., PENA, J.M.; RODRIGO, C.; VIDAL, R. 2001 Prevención de la transmisión vertical y tratamiento de la infección por el virus de la inmunodeficiencia humana en la mujer embarazada. Enfer. Infecc. Microbiol. Clin. 19:314-335.
KOURTIS, A.P.; LEEF, K.; ABRAMS, E.J. 2006. Mother to child transmission of HIV-1: timing and implications for prevention. Lancet Infect Dis. 6:726-732.

KUHN, L.; COUTSOUDIS, A.; TRABATTONI, D.; ARCHARY, D.; ROSSI, T; SEGAT, L.; CLERICI, M.; CROVELLA, S. 2000. HIV-1 specific T-helper cell responses detect al birth. In: Program and abstracts of the seventh conference on retroviruses and opportunistic infections. San Francisco CA 2000. Foundation for retrovirology and human health. Alexandria VA. Abstract 702:206.

LANDESMAN, S.H.; KALISH, L.A.; BURNS, D.N.; MINKOFF, H.; FOX, H.E.; ZORRILLA, C.; GARCÍA, P.; FOWLER, M.G.; MOFENSON, L.; TUOMALA, R. 1996. Obstetrical factors and the transmission of human immunodefiency virus type 1 from mother to child. The Women and Infants Transmission Study. New Engl. J. Med. 334:1617-1623.

MIN. PROT. SOCIAL (COLOMBIA). 2000. Resolución 00412. Por la cual se establecen las actividades, procedimientos e intervenciones de demanda inducida y obligatorio cumplimiento y se adoptan las normas técnicas y guías de atención para el desarrollo de las acciones de protección especifica y detección temprana y la atención de enfermedades de interés en salud publica. Guías de atención No 11: VIH-Sida.

MIN. PROT. SOCIAL. 2003. Dirección General de Salud Pública. Política Nacional de Salud Sexual y Reproductiva. Bogota 2003.

MINKOFF, H.L.; EISENBERGER-MATITYAHU, D.; FELDMAN, J.; BURK, R.; CLARKE, L. 1999. Prevalence and incidence of gynecologic disorders among women infected with human immunodeficiency virus. Am. J. Obste Gynecol. 180:824-836j.

MOR, Z.; CHENTOB, D.; PESSACH, N. 2006. Human immunodeficiency virus in newborn of infected mothers: pregnancy, breast feeding and prevention. Harefuah. 145:682-686.

ONUSIDA. 2002. Programa conjunto de Naciones Unidas sobre VIH/SIDA. Los jóvenes y el VIH/SIDA una 
oportunidad en un momento crucial. ONUSIDA reporte 2002. p.1-9.

ONUSIDA. 2004. Informe sobre la epidemia mundial de Sida 2004.Cuarto informe Mundial. Ginebra. Onusida 2004. p.1-188.

ONUSIDA. 2006. Joint United Nations Programme on HIV/AIDS (UNAIDS) and World Health Organization (WHO). Epidemic update 2003

OPS - ORGANIZACIN PANAMERICANA DE LA SALUD 1999: La mujer y la infección por el VIH/SIDA: Estrategia de prevención y atención. p.1-44.

ORIO, M.; PENA, J.M.; RIVES, M.T.; SANZ, M.; BATES, I., DE MADERO, R.; JOSE, M.I. 2007. Changes in vertical HIV transmission comparison between 1994 and 2004. Med. Clin. 128:321-324.

PÉREZ, F.; DABIS, F. 2003.HIV prevention in Latin America: reaching youth in Colombia. AIDS Care 15:77-87.

PRIETO, F. 2005. Veinte años del VIH en Colombia, 1983-2003.Datos de la vigilancia epidemiológica. Inf. Quinc. Epidem. Nac. 8:355-364.

PROFAMILIA. 2000.Salud Sexual y reproductiva.Resultados de la encuesta Nacional de Demografía y salud 2000. p.73-76

ROUET, F.; EKOUEVI, D.K.; INWOLEY, A.; CHAIX, M.L.; BURGARD, M.; BEQUET, L.; VIHO, I.; LEROY, V.; SIMON, F.; DABIS, F.; ROUZIOUX, C. 2004. Field evaluation of a rapid human immunodeficiency virus (HIV) serial serologic testing algorithm for diagnosis and differentiation of HIV type 1 (HIV-1), HIV-2, and dual HIV-1-HIV-2 infections in West African pregnant women. J. Clin. Microbiol. 42:4147-4153.

SABA, J. 1999. PETRA TRIAL STUDY TEAM: Interim analysis of early efficacy of three short AZT/ 3TC combination regimens to prevent mother to child transmission of HIV-1. The PETRA trial, sixth conference on retroviruses and opportunistic infections. Chicago. USA. p.2-6.

SHETTY, A.K.; MALDONADO, Y. 2001. HIV transmission of perinatal HIV-1 transmission in the United States. Neo Reviews. 2:e83-93.
STRINGER, J.S.; VERMUND, S.H. 1999. Prevention of mother to child transmission of HIV-1. Current Opinion Obst. Gyn. 11:427-434.

THE EUROPEAN MODE OF DELIVERY COLLABORATION. 1999. Elective caesarean section versus vaginal delivery in prevention of vertical HIV-1 transmission a randomized clinical trial. Lancet. 353:1035-1039.

THE INTERNATIONAL PERINATAL HIV GROUP. 1999. The mode of delivery and the risk of vertical transmission of HIV-1: a meta-analysis of 15 prospective cohort studies. New Engl. J. Med. 340: 977-987.

THE PETRA STUDY TEAM. 2002. Efficacy of three short course regimens of zidovudine and lamivudine in preventing early and late transmission of HIV-1 from mother to child in Tanzania South Africa and Uganda: a randomized double-blind placebo controlled trial. Lancet. 359:1178-1186.

URREA-GIRALDO, F.; CONGOLINO, M.I.; HERRERA, H.D. 2006. Sexual behaviours and incidence of sexual and reproductive health programs among poor secondary students and public university students in Cali. Cad. Saude Publica. 22:9-15:

VALDÉS, E.R. 2002. VIH-SIDA y embarazo: Actualización y realidad en Chile. Rev. Chil. Obstet. Ginecol. 67:160-166.

WADE, N.A.; UNADKAT, J.D.; HUANG, S.; SHAPIRO, D.E.; MATHIAS, A.; YASIN, S.; CIUPAK, G.; WATTS, D.H.; DELKE, I.; RATHORE, M.; HITTI, J.; FRENKEL, L.; AMELSON, R.; MITH, M.E.; MOFENSON, L.; BURCHETT, S.K. 2004. Pharmacokinetics and safety of stavudine in HIV-infected pregnant women and their infants: Pediatric AIDS Clinical Trials Group Protocol 332. J. Infect. Dis. 190:2167-2174.

ZAMORANO, J.R.; HIRSCH, T.B. 2004. Infección por virus de inmunodefiencia humana, embarazo y deseo de reproducción. Comité SIDA Pediátrico. Soc. Chilena Pediatría. Rev. Chil. Infectol. 21:208-212.

Recibido: enero 19 de 2007

Aceptado: septiembre 11 de 2007 